\title{
Should we establish standards of care for management of elderly patients with inflammatory bowel disease?
}

\author{
Subrata Ghosh MBBS MD FRCPC FRCP FRCPE, Marietta lacucci MD PhD
}

E derly patients with inflammatory bowel disease (IBD) have poorer outcomes than their younger counterparts with increased inpatient mortality rates, infection rates, lymphoma rates (1), and intolerance to immunosuppressive agents or tumour necrosis factor inhibitors (TNFi) (2). These may lead to hesitancy in prescribing effective immunosuppressive drugs and TNFi to elderly patients, especially in individuals with comorbid conditions. Overuse of corticosteroids, however, has significant deleterious effects in elderly patients including osteoporosis, hypertension, diabetes, ocular complications, infections and postsurgical complications. Some elderly patients with IBD may also have very indolent disease and it is important not to overtreat them.

Several studies, including the report by Stepaniuk et al (3) in the current issue of the Journal (pages 273-277), have clearly shown that elderly patients receive less TNFi and less immunosuppressive drugs than their younger counterparts with IBD (4). While whether such undertreatment contributes to poorer outcome and complications in elderly patients still requires robust evidence, it is also of concern that elderly patients may be treated and monitored less rigorously and objective end points, such as mucosal healing, may never be reached. Introduction of newer therapies, such as the integrin inhibitor vedolizumab, may offer potentially certain safety advantages. TNFi may also be considered in this population as monotherapy rather than combination therapy in the presence of comorbid conditions such as chronic obstructive airways disease. Rapid control of more severe disease is essential to prevent increased morbidity and complications in elderly individuals.

Several areas of management of IBD in elderly patients require specific attention (Box 1). These include establishment of diagnosis by robust exclusion of several conditions such as colon cancer, infections, especially Clostridium difficile, ischemic colitis, diverticular colitis and angiodysplasia. Monitoring by regular use of C-reactive protein, fecal calprotectin, complete blood count and liver function tests is necessary to avoid the necessity of frequent invasive investigations and avoid hematological or other complications. Transabdominal ultrasonography for distal ileal Crohn disease may be a very useful noninvasive monitoring modality because it is well tolerated. Endoscopic surveillance to detect dysplasia may be especially challenging in elderly patients due to the high prevalence of diverticular disease and polypoid lesions leading to difficulty in deciding on local endoscopic resection strategies (5) versus panproctocolectomy with potentially increased morbidity. It is also important to vaccinate elderly patients against common infective preventable diseases and be aware of the high incidence of herpes zoster. The use of steroid-sparing agents, such as azathioprine, methotrexate and $\mathrm{TNFi}$, is important in elderly patients to avoid excessive use of steroids in moderate to severe IBD and ensure mucosal healing. Finally, inpatients need careful prophylaxis against thromboembolism, vigilance against nosocomial infections and surgery if required in a timely manner before advanced disease, poor nutrition and infections lead to postoperative complications and poor outcome including mortality. Elderly patients are often troubled by fecal incontinence after pouch surgery and a permanent ileostomy is often a preferred approach. However, elderly patients with adequate sphincter function may do well after pouch surgery. Multidisciplinary management of these elderly patients is especially important given the comorbid conditions that are often present.

While there are many guidelines and an increasing number of standards of care to optimize management of IBD, there are no specific guidelines for comprehensive management of elderly patients with IBD. This appears to be an omission that requires attention by national and international organizations. Many of the issues in the elderly population also require further generation of evidence because these patients form only a small fraction of patients in clinical trials.

\section{BOX 1 \\ Specific issues influencing management of IBD in elderly patients}

Differential diagnosis: Distinguish among ischemic colitis, colon cancer, diverticular colitis, Clostridium difficile, lymphocytic colitis, angiodysplasia.

Management: Variation in care compared with nonelderly patients, especially lower use of immunosuppressive drugs and TNFi. Risk for poor control of disease.

Complications: Increased risk for infections, lymphoma, herpes zoster, mortality after surgery. Fecal incontinence after ileal-pouch anal anastomosis.

Dysplasia surveillance: Increased prevalence of colonic polypoid lesions and challenge to determine local resection versus panproctocolectomy.

Challenges to therapy: Comorbid conditions such as heart failure, diabetes, hypertension, chronic obstructive lung disease, concurrent or previous malignancy, drug interactions, thromboembolism and osteoporosis.

\section{REFERENCES}

1. Beaugerie L, Brousse N, Bouvier AM, et al. Lymphoproliferative disorders in patients receiving thiopurines for inflammatory bowel disease: aprospective observational cohort study.

Lancet 2009;374:1617-25.

2. Desai A, Zator ZA, de Silva $P$, et al. Older age is associated with higher rate of discontinuation of anti-TNF therapy in patients with inflammatory bowel disease. Inflamm Bowel Dis 2013;19:309-15.

3. Stepaniuk P, Bernstein CN, Nugent Z, Singh H. Characterization of inflammatory bowel disease in hospitalized elderly patients in a large central Canadian health region. Can J Gastroenterol Hepatol 2015;29:273-7.

4. Juneja M, Baidoo L, Schwartz MB, Barrie A, Regueiro M, Dunn M, Binion DG. Geriatric inflammatory bowel disease: Phenotype presentation, treatment patterns, nutritional status, outcomes, and comorbidity. Dig Dis Sci 2012; 57:2408-2415.

5. Iacucci M, Uraoka T, Fort Gasia M, Yahagi N. Novel diagnostic and therapeutic techniques for surveillance of dysplasia in patients with inflammatory bowel disease. Can J Gastroenterol Hepatol 2014;28:361-70.

Department of Medicine, Division of Gastroenterology, University of Calgary, Calgary, Alberta

Correspondence: Dr Subrata Ghosh, Division of Gastroenterology, Department of Medicine, University of Calgary, 3280 Hospital Drive

Northwest, Calgary, Alberta T2N 4N1. Telephone 403-944-8222, fax 403-944-1095, e-mail subrata.ghosh@albertahealthservices.ca Received and accepted for publication April 2, 2015 


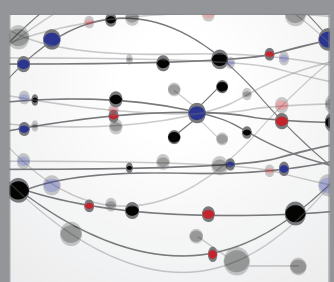

The Scientific World Journal
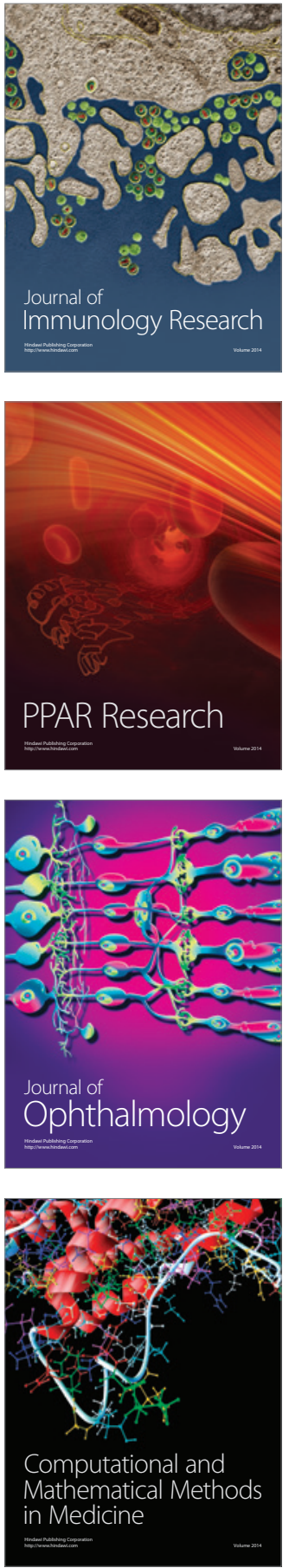

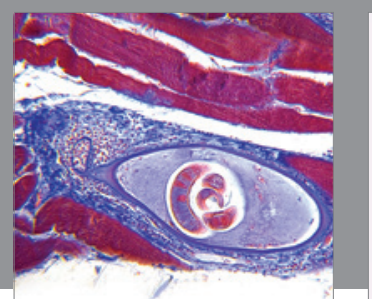

Gastroenterology Research and Practice

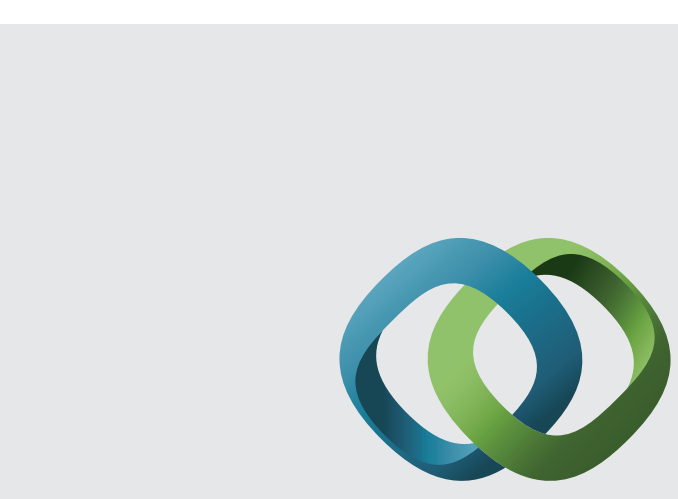

\section{Hindawi}

Submit your manuscripts at

http://www.hindawi.com
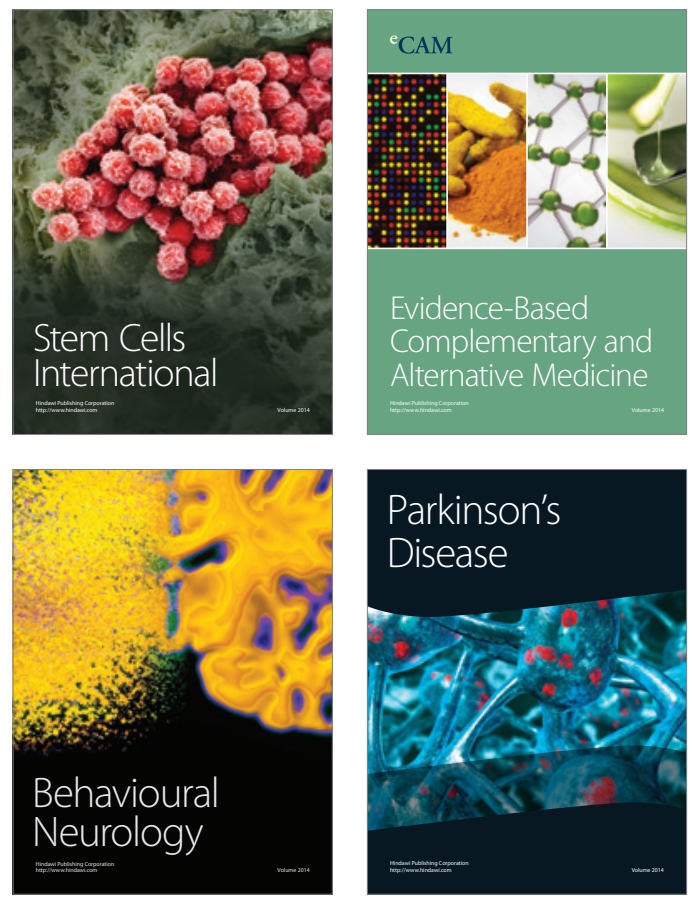
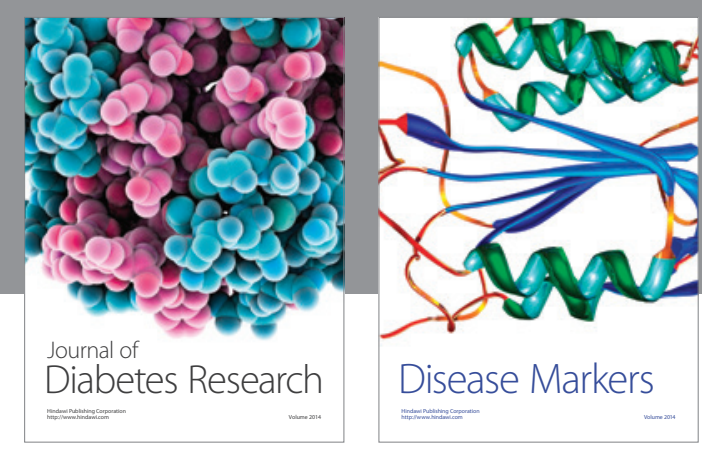

Disease Markers
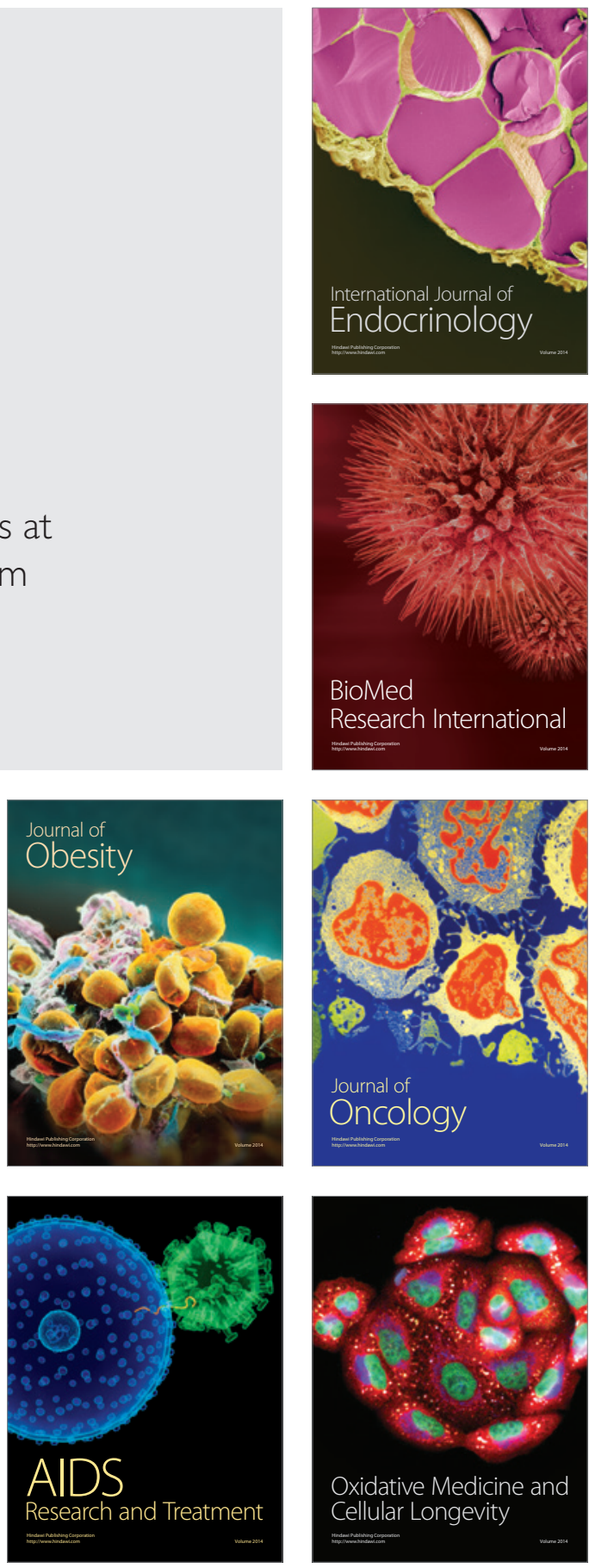